\title{
Thoracic ultrasound in children: reply to Maggi et al
}

\author{
Paolo Tomà • Catherine M. Owens
}

Received: 19 December 2013 / Accepted: 30 January 2014 /Published online: 21 February 2014

(C) Springer-Verlag Berlin Heidelberg 2014

Sir,

We are pleased that the readers [1] found our paper [2] helpful, and we fully agree with the initial part of their Letter to the Editor.

1. Regarding Figure 1, the apparently thicker visceral pleura, in fact, is an artefact due to reflection from the air-containing lung: The visceral pleura is completely obscured by the complete reflection of the ultrasound beam at the lung surface [3]. The parietal pleura is enhanced in a small tract (corresponding to the arrowheads) for artefacts related to respiratory movements and beam incidence.

Concerning the sonographic diagnosis of respiratory distress syndrome and transient tachypnea of the newborn: we agree that they are based on nonspecific signs of lung disease, which originate at the surface of the lung, without complete panoramic views.

2. We also remind the readers of the limitations in the control of various ultrasonographic technical devices, and we believe that chest radiographs are still needed for accurate diagnosis.

3. It is well known that scattered B-lines may be present in normal lung and in the presence of a pneumothorax the fundamental sign to observe is the absence of sliding. Anyway, in pneumothorax in infants, the absence of B-lines is particularly noticeable; hence, the emphasis on the presence of A-lines is more important.

\section{References}

1. Maggi M, Pirri C, Foti T et al (2014) Thoracic ultrasound in children: evidence and drawbacks. Pediatr Radiol 44. doi:10.1007/s00247-0142901-8

2. Toma P, Owens CM (2013) Chest ultrasound in children: critical appraisal. Pediatr Radiol 43:1427-1434

3. Reuss J (2011) Pleura. In: Mathis G (ed) Chest sonography. Springer, Berlin, pp 27-54

P. Tomà $(\bowtie)$

Department of Imaging, Bambino Gesù Children's Hospital, IRCCS,

Piazza S. Onofrio, 00165 Rome, Italy

e-mail: paolo.toma@opbg.net

C. M. Owens

Department of Imaging, Great Ormond Street Hospital for Children,

NHS Trust, London, UK 